\title{
Versteckspiele zwischen Lieferanten und Kunden
}

Verbreitet suchen Unternehmen im Business-to-Business-Vertrieb Lösungen für Kunden anzubieten. Nur verändern sich Lösungen im Laufe der Zusammenarbeit und Lieferanten sowie Kunden spielen oft mit verdeckten Karten.

Christian Belz 
Der Kunde wäht bei der Unterzeichnung von Verträgen oft nur Teile der späteren Lüsung eines Lieferanten. Nach dem Zuschlag entwickeln sich komplexere Projekte und die Anforderungen der Kunden andern sich. Nicht selten wird angestrebt, zusätzliche Leistungen in den ursprünglichen Projeklumfang zu integrieren. Die Lieferanten versuchen ihrerseits, entstehende Leistungen zusätzlich in Rechnung zu stellen. Es gilt deshalb, Leistungspakete als dynamischen Prozess zu gestalten. Begleitet wird die Zusammenarbeit durch laufende Verhandlungen. Gleichzeitig gilt es, mit dem Versteckspiel zwischen Lieferanten und Kunden wirksam umzugehen.

Diese Hinweise betreffen den Vertrieb im Zusammenspiel mit den weiteren Kundenkontaktpersonen in Technik, Logistik, Informatik und Kundendienst. Ebenso wechselt die Zuständigkeit bei Kunden.

Der folgende Fall beschreibt die Ausgangslage: Y.AG entwickelt und produziert Produkte für andere Unternehmen. Für einen Großkunden der Lebensmittelindustrie industrialisierte das Unternehmen eine komplexere Produktfamilie von Küchengeräten und erhielt den Zuschlag für die Serienfertigung. Dieser Auftrag ist bedeutend. Allerdings bringt der Kunde verschiedene, zusätzliche Ansprüche ein. Sie betreffen beispielsweise die Logistik, Produktanpassungen oder die Einrichtung eines Inbound Marketings für Anliegen der Konsumenten. Für diese Aufgaben ist Y.AG qualifiziert. Nur will der Kunde für zusätzliche Aufgaben nicht bezahlen und betrachtet sie als Bestandteil des langfristigen Produktionsauftrags. Generell sind die Anforderungen an Teilequalität, Produktionsmittel, Reaktionsgeschwindigkeit, Kundensupport und Auftragsabwicklung sehr hoch. Mit den Zusatzanforderungen des Kunden droht das attraktive Projekt für Y.AG in die Verlustzone zu gleiten.

\section{Prozess der Zusammenarbeit}

Komplexere Angebote gehen immer von Annahmen aus. Im Laufe der Zusammenarbeit ändern sich die Erkenntnisse und Anforderungen der Kunden, aber ebenso der Lieferanten. Ein rigoroser Preisdruck in der Vergabephase vermindert spätere Spielräume. Wenn erste Projektschritte erfolgreich vollzogen wurden, entstehen neue Aufgaben, um das ganze Projekt zum Erfolg zu führen.

Zwar vermuten Kunden und Anbieter, dass spater etwas auf sie zukommt. Nur sind beide Parteien bedingt motiviert, die Folgeaufgaben alle einzubeziehen oder abzugrenzen. Die Verantwortlichen des Kunden befürchten, dass das Projekt intern scheitert, wenn bereits zu viele Folgen einbezogen werden. Der Lieferant will das Projekt gewinnen und stellt beispielsweise auch kritische Aspekte in einer Ausschreibung des Kunden nicht in Frage. Deshalb ist es wohl auch blauäugig, für das Gesamtprojekt und den Gesamtvertrag bereits eine umfassende Transparenz zu fordern, die Leistungen klar zu spezifizieren und auch zu begrenzen.

Begleitet wird ein solcher Prozess durch die unterschiedliche Spannung des Lieferanten und des Kunden im Zeitablauf. Während der Verkäufer sein Ziel und Höhepunkt mit dem Kaufabschluss erreicht, setzen die hohen An-

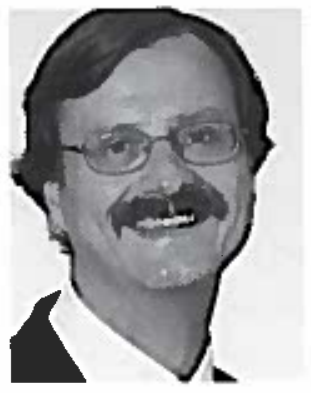

Prof. Dr. Christian Belz ist Ordinarius für Marketing un der Universität St. Gallen und Direktor amt Institut fär Marketing.

Christian Belz

Universität St. Gallen, St. Gallen, Schweiz

E-Mail: christian.belz unisg.ch 
sprüche des Kunden erst nach dem Kaufentscheid richtig ein. Diese Zusammenhänge zeigte der klassische Artikel von Theodore Levitt zu, After the Sale is Over' (Harvard Business Review 9/1983) bereits deutlich. Deshalb schlägt dic Großzügigkeit des Lieferanten in der Gewinnungsphase für ein Projekt nach dem Kauf oft um, in die Abwehr oder konsequente Verrechnung von Zusatzleistungen. Nicht selten vertraut der Anbieter darauf, dass er ein gewonnenes Projekt mit Preisdruck im Laufe der Zusammenarbeit in einer weiteren $\mathrm{Zu}$ sammenarbeit fïr sich verbessern kann.

\section{Die Prozesse im einfachen Modell}

Die beschriebenen Prozesse lassen sich grob in Abbildung 1 darstellen. Die obere Hälfte der Abbildung zeigt Haupt- und Nebenprojekte im Zeitablauf. Jedes dieser Projekte schließt ein:
- verschiedene Phasen von Initialphase, Evaluation, Konzeption bis Realisierung (dabei sind Phasen für konkrete Projekto auch spezifisch definiert)

- Verlauf von internen und externen Kosten des Lieferanten und des Kunden

- Verlauf des „Zusammenarbeitsklimas“

- unterschiedliche Beteiligte im Anbieter- und Kundenunternehmen je nacl Phase

- mehrere Schtüsselstellen für Zwischenentscheide

In einem schrittweisen Prozess (untere Hälfte der Abbildung) handeln Kunde und Lieferant aus, welche Leistungen im Hauptprojekt integriert sind und was zusätzlich belastet werden kann. Dominiert das Interesse des Kunden, so verwandelt sich das Hauptprojekt des Lieferanten in ein Verlustgeschäft. Dominiert das Interesse des Anbieters, überborden

\section{Abb. 1 Dynamische Leistung und Verhandlung}

Nebenprojekt 1 (z.B. Logistik)

\section{Hauptprojekt (z.B. Serienfertigung eines Produktes)}

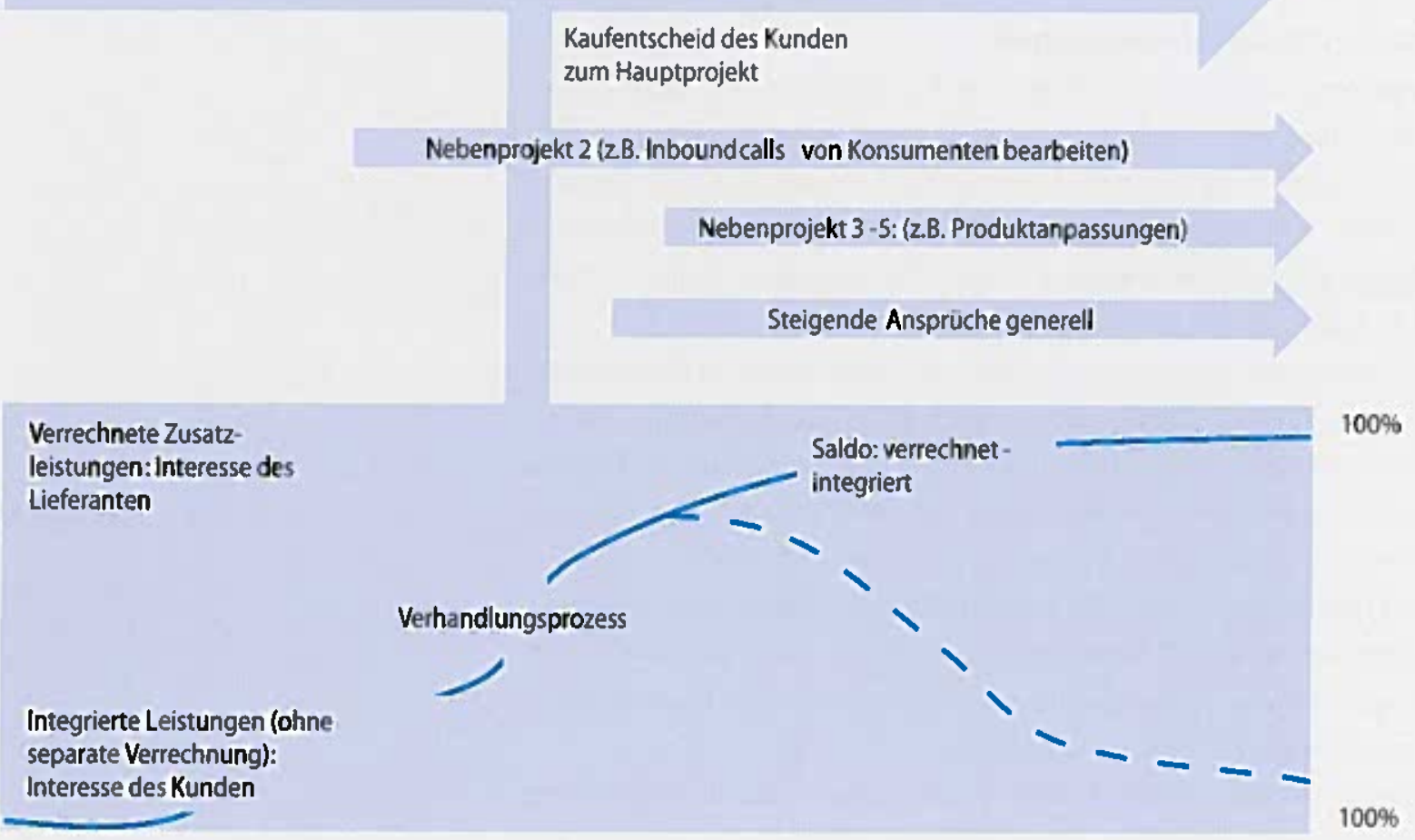

Quelle: eigene Darstellung 
die Kosten für den Kunden in seinem Hauptprojekt und den Zusatzleistungen.

Beide Phänomene lassen sich beobachten. Ersteres ist durch die Machtposition der Kunden häufiger. Letzteres trifft ein, wenn ein Kunde durch die Vorentscheide abhängig wird. Die Varianten werden durch gestrichelte und ausgezogene Linie gezeigt.

\section{Beide Parteien arbeiten mit Vermutungen}

Die folgenden Hinweise verdanke ich Dr. Michael Reinhold von der Universität St. Gallen: In einer Zusammenarbeit decken Kunden ihre Ansprüche nicht völlig auf und auch Lieferanten halten ihre Absichten teilweise zurïck. Manches ist einfach nicht bewusst, anderes beruht auf konkurrierenden Zielen. Unter diesen Voraussetzungen lässt sich das Zusammenspiel nur begrenzt optimieren, beide Parteien arbeiten dann mit Vermutungen. Angebote können in dieser Konstellation falsch liegen, ohne dass die Gründe dafür bekannt sind. Aus Anbietersicht interessieren besonders die verdeckten Bedürfnisse und Auswahlkriterien des Kunden:

Auch das Versteckspiel betrifft nicht nur die große Auftragsvergabe, sondern begleitet sämtliche expliziten und impliziten Teilverhandlungen im Prozess der Zusammenarbeit.

Um den Kundennutzen zu steigern, richten Anbieter ihre Leistung besser auf Bedürfnisse des Kunden (oder von Kundengruppen) aus, sie verzichten auf Unnötiges und erweitern ihr Angebot. Sie können auch versuchen, dem Kunden zu zeigen, dass ihre Leistung wichtigen Bedürfnissen entspricht, die sie bisher nicht erkannten. Damit bewegen sie den Kunden zu

\section{Zusammenfassung}

- Im Laufe einer anspruchsvollen Zusammenarbeit von Anbietern und Kunden verändern sich die Lösungen laufend. Nach dem Kauf folgen weitere Zwischenschritte mit Leistungen und Gegenteistungen, die ein Projekt zum Erfolg oder Misserfolg führen können.

- Das Verhältnis zwischen Lieferanten und Kunden ist nicht transparent. Zielkonflikte, sachliche Gründe oder Machtausübung sind die Gründe.

- Es gilt, Lösungen als Prozess zu führen und nach dem Kaufentscheid besonders wachsam zu agieren.

- Wichtig ist es, die Versteckspiele zu akzeptieren und auszuloten.

ihrer Leistung. Natürlich spielt dabei auch der Vergleich zum Angebot der Wettbewerber eine Rolle.

Sowoht Bedürfnisse als auch Angebote sind jedoch nicht alle transparent und werden in der Zusammenarbeit offengelegt.

Manches ist dem Kunden einfach selbst nicht bekannt. Mögliche Voraussetzungen für eine Beschaffung, zusätzliche Kriterien oder vernachlässigte und schlicht unterschätzte Bedürfnisse lassen sich dann zwischen Anbieter und Kunden besser austoten. Allerdings hat der Anbieter meist nur das Interesse, jene Bedürfnisse zu klären, die er auch abdecken kann, was der Idee eines Value Selling entgegengesetzt ist.

\section{Abb. 2 Kundennutzen als Schnittmenge zwischen Kundenbedürfnissen und Angebot}

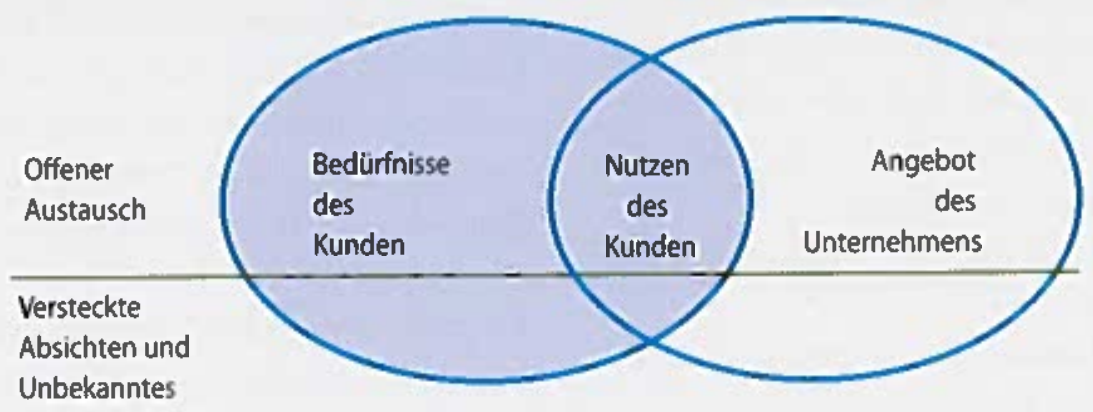

Quelle: Erweiterte Darstellung nach Weinhold, $H_{4}$ : Marketing in 20 Lektionen, Heerbrugg: Fachmed 1991, S. 192 
Anbieter und Kunden hiben auch unterschiedliche Ziele, offensichtlich ist der Anbieter an hohen Preisen interessiert, wahrend der Kunde den tiefen Preis anstrebt; das betrifft alle Leistungen und Gegen]eistungen. Um die eigenen Ziele zu erreichen, spielen beide mit verdeckten Karten. Sie halten Informationen zurück. Das außert sich auch in Verhandlungen. So kann der Kunde begeistert sein oder er ist abhängig vom Lieferanten, lisst ihn das aber möglichst wenig spüren und gibt sich im Gegenteil kritisch. Beispielsweise erkennt der Anbieter verschiedene Folgeprobleme einer Anschaffung oder spätere Kosten des Kunden, möchte dies aber nicht thematjsieren, um seine Position nicht zu schwächen. Auch erkennt der Verkäufer eventuell in Diskussionen, Unterlagen oder beim Durchgang durch die Produktion des Kunden verschiedene Lösungen, die er dann beim Wettbewerber des Kunden im nächsten Gespräch nutzen will.

Natürlich gibt es in diesem Wechselspiel auch ethische Grenzen. Hier interessiert mehr, was der Kunde versteckt und nicht der Lieferant. Misstrauen und Verschlossenheit steigern sich jedoch gegenseitig. Warum sagt der Kunde nicht, was er wirklich will? Es gibt verschiedene Gründe, weshalb der Kunde nicht alles mitteilt, was er will. Einige Beispiele:

- Versteckte Strategie: Eine Beschaffung hängt mit einer neuen Technologie oder/und einem neu entdeckten Markt des Kunden zusammen. Der Kunde will diese Strategie nicht offenlegen, weil damit die Branche durch die Lieferanten frühzeitig informiert werden kônnte.

\section{Kerngedanken}

- Gefeierte Zuschläge des Kunden können nach dem Kauf zum Verlustgeschäft umschlagen. Der Preisdruck für ein Angebot verlagert die Anstrengungen für gute Geschäfte.

- Mit einem üblichen Projektmanagement lässt sich den wechselnden Ansprüchen der Kunden und neuen Erkenntnissen der Lieferanten nicht allein beikommen. - Die Zielkonfikte zwischen Lieferant und Kunde führen zu einem natürlichen Versteckspiel.

- Kostentransparenz ist wichtige Voraussetzung für eine vernünftige Diskussion.

- Die Zusammenarbeit mit Kunden stützt sich auf periodische oder laufende Verhandlungen.
- Versteckte Konzepte: Eigene Konzepte und Kriterien werden nicht offengelegt, weil der Kunde bei lieferanten prüen wilt, ob sie auf die gleichen Lösungen stoßes.

- Know-how-Abfluss: Der Kunde zeigt seine Lösungen und die Integration der Beschaffungsgüter nicht, damit dieses Know-how nicht zu den eigenen Wettbewerbern weitergetragen wird.

- Eigenes Engagement: Der Kunde sucht die Zusammenarbeit mit einem Lieferanten, um von ilim zu lernen und die Zeit zu überbrücken, bis er die entsprechenden Leistungen selbst erbringt. Befürchtet der Lieferant diese zukünftige Konkurrenzierung durch den Kunden, so sucht er sie zu erschweren. Teilweise arbeiten Kunden und ihre Lieferanten an ähntichen Projekten und stehen damit gleichzeitig in Konkurrenz. Sie soll nicht of fengelegt werden.

- Versteckte und divergierende Agenda im Buying Center: Versteckt bleiben oft die verschiedenen Prioritäten von Entscheidern und Beeintlussern. So können Grundsatzüberlegungen der Geschäftsleitung selbst intern den einkaufenden Personen unbekannt sein. Auch intern wird (aus ähnlichen Gründen wie im Verhältnis von Lieferanten und Kunden) oft verdeckt argumentiert.

- Abschirmung: Die Kontaktpersonen blockieren die Kontakte zu weiteren Personen im Unternehmen und streichen ihre eigene Bedeutung heraus.

Es gibt durchaus sachliche Gründe für Kunden, nicht alles zu sagen. Andere Gründe hängen mit der Demonstration von Macht bis zu unbegründeten Ängsten, Misstrauen oder mangetndem Engagement zusammen. Teilweise verschwenden Kunden auch einfach die Ressourcen ihrer potenzietlen Partner. Manchmal weiß der Kunde, was er braucht, aber verantwortliche Personen akzeptieren das nicht oder sind dafür nicht zuständig.

Analog wissen oft Anbieter, dass ihre Kunden etwas brauchen, sie verkaufen jedoch in der vorgegebenen Konstellation des Kunden etwas Falsches. Ob sie die Verhältnisse dem Kunden nur andeuten oder gar eine Lieferung verweigern, sch tießt viele Reaktionen ein. Die versteckte Agenda des Kunden führt oft dazu, dass Lieferanten in Unkenntnis der Verhältnisse ein falsches Angebot abgeben.

\section{Empfehlungen für Kunden und Lieferanten}

Drei Empfehlungen lassen sich für Kunden und Lieferanten wie folgt zusammenfassen:

1. Projektklärung: Bei repetitiven Aufgaben für Kunden lässt sich eine Transparenz zut Zusammenarbeit zum Zeitpunkt 
den verschiedenen Prozessen. Falsche Gesprächspartner führen zu falschen Frgebnissen.

\section{Gemeinsame Interessen im Vordergrund}

Wichtig bleibt dabei, sich nicht auf eigene Positionen zu verfestigen, sondern im Sinne einer Mediation die gemeinsanen Interessen in den Vordergrund zu stellen. Emotional braucht es gegenseitige Akzeptanz und Anstand, ein konstruktives Klima, Fairness und Vertrauen. Das Misstrauensprinzip scheint sich zwar in der Wirtschaft mehr und mehr auszudehnen, verhindert aber Hochleistungen systematisch.

Ergebnis der Verhandlungen sind im Kern die Leistungsbegrenzungen oder-erweiterungen sowie die Verteilung von Kosten und Erträgen zwischen Lieferanten und Kunden. Daraus ergibt sich ein dynamischer Verrechnungsprozess.\#

Im Umgang mit dem Versteckspiel sind zudem folgende Möglichkeiten zu prüfen:

- Die offene Zusammenarbeit kann als Voraussetzung für Kunden- und Projektselektion dienen. Dabei spielen auch frühere Erfahrungen mit dem Kunden eine Rolle.

- Die Grenzen der Lösungsangebote durch Kunden gilt es zu akzeptieren und gleichzeitig ein Sensorium dafür zu entwickeln.

- Die versteckten Aspekte zur Zusammenarbeit gilt es aktiv zu suchen.

- Je nach vermuteten Hürden lassen sich spezifische Lösungen prüfen. ,Non disclosure agreements' oder Vereinbarungen zur Geheimhaltung lösen die Problematik aber kaum. So könsen ja Lieferanten bei Kunden auf Hinweise und Lösungen stoßen, die sie bereits kennen oder selbst entwickeln.

Geheimnisse in der Zusammenarbeit sind normal. Bemühen sich jedoch Lieferanten und Kunden darum, mogliche Lücken für den Partner vernünftig zu überbrücken, so lassen sich schon viele Fehlleistungen verhindern. Diese Hinweise sind noch recht generisch. Konkrete Projektverläufe und spezifische Geheimnisse prägen die konkreten Chancen und Ge fahren in der Zusammenarbeit. Deshalb ist es notwendig, die spezifischen Prozesse zu gestalten.

\section{Fazit}

Lösungen für Kunden werden in der Forschung und auch Pra$x$ is mehrheitlich statisch erfasst. Anbieter definieren ein Leistungspaket und der Kunde wählt die passenden Bausteine. Besonders für komplexere Leistungen müssen wir aber zukünftig die Dynamik der Zusammenarbeit besser durchdringen. Natürlich ist ein rigoroses Projektmanagement in einer umfangreichen Zusammenarbeit naheliegend. Aufwand und Kosten müssen geführt werden, damit sich Projekte nicht verselbstständigen. Prozessorientierte Lösungen und dynamische Verrechnung gilt es jedoch, explizit zu erfassen, um den realistischen und erfolgreichen Umgang mit dem Lösungsgeschäft zu fördern. Die Wahl eines Anbieters oder eines Kunden ist erst die Einstiegskarte. Wichtiger ist, wie später die Zusammenarbeit gelebt wird. Begleitend gilt es auch zu berücksichtigen, dass weder Kunden noch Lieferanten ihre Absichten offenlegen.

Zusätzlicher Verlagsservice für Abonnenten von „Springer für Professionals | Vertrieb“

Zum Thema Kundenlösungen

\section{Medlum}

[ On'ine-Artikel ( 3 )

प Zeitschriftenartikel (9)

$\square$ Buchkapitel (22)

Sprache

$\square$ Deutsch (34)
Von der Verlagsredaktion empfohlen

Schmitz, G.: Der kundenseitig wahrgenommene Beziehungswert - Eine empirische Analyse im Kontext von Privatkundenlösungen, in: Schmitz, G: Service Value als Wertreiber, Wiesbaden 2014, S. 137-160, www.springerprofessional.de/5063574

Kordowich, $P$.: Auswirkungen von Kundenlösungen auf die Kommunikationsbeziehumgen, in: Kordowich, P.: Betrieblich Kommunikationsprozesse bei Dienstleistern, Wiesba den, Wiesbaden 2010, S. 143-174, www.springerprofessional.de/1844822 